\title{
Convergence analysis of finite element approximations of the Joule heating problem in three spatial dimensions
}

\author{
Michael J. Holst • Mats G. Larson • Axel Målqvist • \\ Robert Söderlund
}

Received: 24 April 2010 / Accepted: 9 September 2010 / Published online: 29 September 2010

(C) The Author(s) 2010. This article is published with open access at Springerlink.com

\begin{abstract}
In this paper we present a finite element discretization of the Joule-heating problem. We prove existence of solution to the discrete formulation and strong convergence of the finite element solution to the weak solution, up to a sub-sequence. We also present numerical examples in three spatial dimensions. The first example demonstrates the convergence of the method in the second example we consider an engineering application.
\end{abstract}

Keywords Finite element methods - Joule heating problem · Convergence analysis

Mathematics Subject Classification (2000) 65 N30 $\cdot 35 \mathrm{~J} 60$

Communicated by Ralf Hiptmair.

A. Målqvist work was supported by The Göran Gustafsson foundation.

M.J. Holst

Department of Mathematics, University of California at San Diego, La Jolla, CA, 92093-0112, USA

e-mail:mjhsan@ccom.ucsd.edu

M.G. Larson · R. Söderlund

Department of Mathematics, Umeå University, 90187 Umeå, Sweden

M.G. Larson

e-mail: mats.larson@math.umu.se

R. Söderlund

e-mail: robert.soderlund@math.umu.se

A. Målqvist ( $\varangle)$

Department of Information Technology, Uppsala University, 75105 Uppsala, Sweden

e-mail: axel.malqvist@it.uu.se 


\section{Introduction}

In this paper we study the stationary Joule heating problem,

$$
\begin{cases}-\nabla \cdot(\sigma(u) \nabla \phi)=f & \text { in } \Omega, \\ \phi=0 & \text { on } \partial \Omega, \\ -\Delta u=\sigma(u)|\nabla \phi|^{2} & \text { in } \Omega, \\ u=0 & \text { on } \partial \Omega,\end{cases}
$$

where $\Omega \subset \mathbf{R}^{3}$ and $\sigma$ is a bounded positive function. The system models interaction between temperature $u$ and electric potential $\phi$ in a device. The minimal assumption to get a finite energy solution in the potential equation, i.e. $\phi \in H_{0}^{1}(\Omega)$, given a fixed $\sigma(u)$, is $f \in H^{-1}(\Omega)$. However, if we only have finite energy in the potential equation, which is the most common in the literature see e.g. $[4,8]$, then the right hand side of the heat equation will reside $L^{1}(\Omega)$. In three spatial dimensions this does not lead to finite energy solutions i.e. $u \notin H_{0}^{1}(\Omega)$.

In the recent work [3] it is shown, under the additional assumption that $\phi$ is bounded, that in fact the temperature has finite energy. The proof is based on the following identity

$$
\sigma(u)|\nabla \phi|^{2}=f \phi+\nabla \cdot(\phi \sigma(u) \nabla \phi),
$$

which holds in the sense of distribution, and the assumptions $f \in L^{\infty}(\Omega), \sigma(u) \in$ $L^{\infty}(\Omega)$, and that the domain is Lipschitz, see Lemma 2.3 below. We will use the alternative right hand side (1.2) together with standard continuous piecewise linear finite elements to construct the finite element approximation. The main result of this paper is a proof of strong convergence of the finite element approximation in $H_{0}^{1}(\Omega) \times H_{0}^{1}(\Omega)$, up to a sub-sequence, under the additional assumption that the discretization preserves the max norm bound in the approximation of $\phi$. We also construct and implement a family of meshes in three spatial dimension for which the discrete maximum principle for the potential equation holds. This has not been done before for the Joule heating problem. Note that under these assumptions on the continuous problem an exact solution $(\phi, u)$ is only in $H_{0}^{1}(\Omega) \cap L^{\infty}(\Omega) \times H_{0}^{1}(\Omega)$ and thus the result presented here is significantly different from standard a priori error analysis of finite element methods which typically relies on stronger regularity of the exact solution. For results of similar type we refer to $[4,5]$ where numerical methods for problems with $L^{1}(\Omega)$ data are presented. Here less regularity is assumed in the trail space but instead the test functions are assumed to be continuous. We present two numerical examples in three spatial dimensions. First we consider a model problem and study convergence and then we apply the method to a more realistic engineering application, Joule heating in a MEMS (Micro Electro Mechanical System) device. For more information on the Joule heating problem and in particular the time dependent version of the problem see [2, 7].

The reminder of the paper is organized as follows. In Sect. 2 we present the continuous problem and prove existence of solution. In Sect. 3 we present the discrete problem and prove existence of solution. In Sect. 4 we prove convergence of the discrete approximation to the weak solution and, finally, in Sect. 5 we present the numerical examples. 


\section{The continuous problem}

Before we give a more precise definition of the continuous problems we need to introduce some notation.

\subsection{Preliminaries}

We let $\Omega \subset \mathbf{R}^{3}$ be a domain with Lipschitz boundary $\partial \Omega$. We denote by $L^{p}(\Omega)$, for $1 \leq p<\infty$ the set of functions satisfying $\int_{\Omega}|f|^{p} d x<\infty$ and we let $L^{\infty}(\Omega)$ denote the set of functions that are bounded almost everywhere in $\Omega$. Further we let $H^{1}(\Omega)$ denote the space of functions that are in $L^{2}(\Omega)$ and that has gradients in $L^{2}(\Omega)^{3}$. We note that $L^{2}(\Omega)$ and $H^{1}(\Omega)$ are Hilbert spaces and we introduce the following notation for the inner product in $L^{2}(\Omega),(v, w)=\int_{\Omega} v w d x$. Since we consider Dirichlet boundary conditions in this work it is natural to introduce the space $H_{0}^{1}(\Omega)=\left\{v \in H^{1}(\Omega): \operatorname{tr}(v)=0\right\}$, where $\operatorname{tr}(v)$ denotes the trace of the function $v$ on the boundary $\partial \Omega$. We let $H^{-1}(\Omega)$ denote the dual space of $H_{0}^{1}(\Omega)$ and extend the definition of $(v, w)$ to also cover the dual pairing between $H_{0}^{1}(\Omega)$ and $H^{-1}(\Omega)$. For an extensive overview of these spaces we refer to [1]. Throughout this paper constants independent of the mesh size will be denoted $C$. Sometimes $C_{i}$ will be used to denote a specific constant that is used several times in the paper.

\subsection{Problem formulation}

We first collect all assumptions on the data and the domain,

$$
\text { (A1) } \begin{cases}f \in L^{\infty}(\Omega), & \\ 0<\alpha \leq \sigma(u) \leq \beta, & \text { where } \alpha, \beta \in \mathbf{R}^{+}, \\ \sigma(u) & \text { is continuous in } u, \\ \Omega \subset \mathbf{R}^{3}, & \partial \Omega \text { Lipschitz boundary. }\end{cases}
$$

From now on we refer to this collection of assumptions as assumption (A1). We multiply both equations in (1.1) by test functions $v, w \in H_{0}^{1}(\Omega)$, and use Green's formula to get the following weak form, find $(\phi, u) \in H_{0}^{1}(\Omega) \times H_{0}^{1}(\Omega)$ such that

$$
\begin{array}{r}
(\sigma(u) \nabla \phi, \nabla v)=(f, v), \quad \text { for all } v \in H_{0}^{1}(\Omega), \\
(\nabla u, \nabla w)=\left(\sigma(u)|\nabla \phi|^{2}, w\right), \quad \text { for all } w \in H_{0}^{1}(\Omega) .
\end{array}
$$

Here the term $\left(\sigma(u)|\nabla \phi|^{2}, w\right)$ only makes sense if $\sigma(u)|\nabla \phi|^{2} w \in L^{1}(\Omega)$. This result is not obvious but it was proven in Lemma 6.1 in [4] using the fact that $\sigma(u)|\nabla \phi|^{2} \geq 0$. This means that, using (1.2),

$$
\left(\sigma(u)|\nabla \phi|^{2}, w\right)=(f \phi, w)+(\nabla \cdot(\phi \sigma(u) \nabla \phi), w), \quad \text { for all } w \in H_{0}^{1}(\Omega) .
$$

We will use this equality to reformulate (2.3) below. We will also show that given $u \in L^{2}(\Omega)$ it holds that $\phi \in H_{0}^{1}(\Omega) \cap L^{\infty}(\Omega)$, in (2.2), using the Lax-Milgram theorem and a max norm estimate. The following Lemma guarantees that (2.3) rewritten 
using (2.4) is well posed if $u \in L^{2}(\Omega)$ and $\phi \in H_{0}^{1}(\Omega) \cap L^{\infty}(\Omega)$ in the right hand side are given.

Lemma 2.1 Given $u \in L^{2}(\Omega), f \in L^{\infty}(\Omega)$, and $\phi \in H_{0}^{1}(\Omega) \cap L^{\infty}(\Omega)$ consider,

$$
\begin{cases}-\Delta \bar{u}=f \phi+\nabla \cdot(\phi \sigma(u) \nabla \phi) & \text { in } \Omega, \\ \bar{u}=0 & \text { on } \partial \Omega,\end{cases}
$$

where $\partial \Omega$ is a Lipschitz domain. There exist a unique solution to (2.5) and the following bound holds,

$$
\|\bar{u}\|_{H^{1}(\Omega)} \leq C\|f\|_{L^{\infty}(\Omega)}\|\phi\|_{L^{\infty}(\Omega)}+C\|\phi\|_{L^{\infty}(\Omega)}\|\sigma(u)\|_{L^{\infty}(\Omega)}\|\nabla \phi\|_{L^{2}(\Omega)} \leq C_{1},
$$

where $C$ depends on the computational domain $\Omega$ but is independent of the solution $\bar{u}$ and the data $f, \phi, u$, and $\sigma$.

Proof The first term in the right hand side of (2.5) does not lead to any problems. Theorem 1.5 in [13] can be applied on the second term in the right hand side, since $\phi \sigma(u) \nabla \phi \in L^{2}(\Omega)$. The Lemma finally follows by using the Poincare-Friedrich inequality.

We use (2.4) to rewrite (2.2) and (2.3) to the equivalent form: find $(\phi, u) \in$ $H_{0}^{1}(\Omega) \cap L^{\infty}(\Omega) \times H_{0}^{1}(\Omega)$ such that,

$$
\begin{aligned}
& (\sigma(u) \nabla \phi, \nabla v)=(f, v), \quad \text { for all } v \in H_{0}^{1}(\Omega), \\
& (\nabla u, \nabla w)=(f \phi+\nabla \cdot(\phi \sigma(u) \nabla \phi), w), \quad \text { for all } w \in H_{0}^{1}(\Omega) \text {. }
\end{aligned}
$$

If $\phi \in H_{0}^{1}(\Omega) \cap L^{\infty}(\Omega)$ and $u \in L^{2}(\Omega)$ are given in the right hand side of (2.8), Lemma 2.1 guarantees that there exist a solution $u \in H_{0}^{1}(\Omega)$. We are going to use this result then constructing a fixed point iteration. We can rewrite the right hand side in (2.8) once again by using Green's formula and that $\operatorname{tr}(w)=0$ we get,

$$
\begin{aligned}
(\sigma(u) \nabla \phi, \nabla v)=(f, v), & \text { for all } v \in H_{0}^{1}(\Omega), \\
(\nabla u, \nabla w)=(f \phi, w)-(\phi \sigma(u) \nabla \phi, \nabla w), & \text { for all } w \in H_{0}^{1}(\Omega) .
\end{aligned}
$$

We are now ready to prove existence results for the weak form, (2.9) and (2.10).

\subsection{Preliminary result for the potential equation}

We fix $u \in L^{2}(\Omega)$ and consider the problem, find $\phi \in H_{0}^{1}(\Omega)$ such that,

$$
(\sigma(u) \nabla \phi, \nabla v)=(f, v), \quad \text { for all } v \in H_{0}^{1}(\Omega) .
$$


The existence of a unique solution $\phi$ to this problem given $u$ follows immediately by using Lax-Milgram's theorem. We also get the standard bound,

$$
\|\phi\|_{H^{1}(\Omega)} \leq C\|f\|_{H^{-1}(\Omega)} \leq C\|f\|_{L^{\infty}(\Omega)},
$$

where $C$ depends on $\alpha$ and $\Omega$. Furthermore, it can be proven that the solution $\phi$ is bounded.

Lemma 2.2 The solution to (2.11) is bounded almost everywhere, i.e. $\phi \in L^{\infty}(\Omega)$ and furthermore $\|\phi\|_{L^{\infty}(\Omega)} \leq C\|f\|_{L^{\infty}(\Omega)}$, where $C$ is independent of $u$.

Proof See e.g. [12].

2.4 Preliminary results for the temperature equation

Given any $\phi \in H_{0}^{1}(\Omega) \cap L^{\infty}(\Omega)$ and $u \in L^{2}(\Omega)$ we state the following weak form, find $\bar{u} \in H_{0}^{1}(\Omega)$ such that,

$$
(\nabla \bar{u}, \nabla w)=(f \phi, w)-(\phi \sigma(u) \nabla \phi, \nabla w), \quad \text { for all } w \in H_{0}^{1}(\Omega)
$$

In the light of equation Lemma 2.1 we immediately get existence of unique solution to (2.13) such that $\|\bar{u}\|_{H^{1}(\Omega)} \leq C_{1}$.

\subsection{Existence of solution to the continuous problem}

Together (2.11) and (2.13) defines a map $\tilde{T}: L^{2}(\Omega) \rightarrow H_{0}^{1}(\Omega)$ i.e. $\bar{u}=\tilde{T} u$. Since $H_{0}^{1}(\Omega)$ is compactly imbedded in $L^{2}(\Omega)$ there is a compact imbedding operator $E$ : $H_{0}^{1}(\Omega) \rightarrow L^{2}(\Omega)$ that identifies an element in $H_{0}^{1}(\Omega)$ with its corresponding element in $L^{2}(\Omega)$. We define the map $T=E \circ \tilde{T}: L^{2}(\Omega) \rightarrow L^{2}(\Omega)$. If we can prove that $T$ has a fix point there will at least exist one solution to (2.9) and (2.10) and therefore at least one weak solution to (1.1).

Lemma 2.3 Under the assumption (A1) there exist a weak solution to (1.1) such that $\phi \in H_{0}^{1}(\Omega) \cap L^{\infty}(\Omega)$ and $u \in H_{0}^{1}(\Omega)$.

Proof This Lemma is proven in [3], see Lemma 2.1. Below we give a variation of the proof for completeness.

Since we have a bound of $\bar{u}=\tilde{T} u$ in $H_{0}^{1}(\Omega)$ independent of $u$ and $\phi$ we have a map $\tilde{T}$ which is bounded. Furthermore, we have that $T$ maps a convex set $S=\{v \in$ $\left.L^{2}(\Omega):\|v\|_{L^{2}(\Omega)} \leq C_{1}\right\}$ into itself. If we can show that $T$ is continuous and compact we can apply Schauder's fixed point theorem to guarantee existence of solution. We have that $T=E \circ \tilde{T}, \tilde{T}$ is bounded and $E$ is compact which means that $E \circ \tilde{T}$ maps bounded sets into pre-compact sets. It remains to prove that $T$ is continuous. 
We assume that we have a sequence $u_{n} \rightarrow u$ strongly in $S$. This means that the corresponding sequence $\phi_{n} \rightarrow \eta$ weakly in $H_{0}^{1}(\Omega)$, for some $\eta \in H_{0}^{1}(\Omega)$, since it is bounded by (2.12). Since $\sigma$ is continuous in $u$, we get $\sigma\left(u_{n}\right) \rightarrow \sigma(u)$ a.e. in $\Omega$. By the Dominated Convergence Theorem $\sigma\left(u_{n}\right) \nabla v \rightarrow \sigma(u) \nabla v$ strongly in $L^{2}(\Omega)$ i.e.

$$
(f, v)=\left(\sigma\left(u_{n}\right) \nabla \phi_{n}, \nabla v\right) \rightarrow(\sigma(u) \nabla \eta, \nabla v), \quad \text { for all } v \in H_{0}^{1}(\Omega) .
$$

Since the solution to (2.11) is unique we conclude $\phi_{n} \rightarrow \phi$ weakly in $H_{0}^{1}(\Omega)$ which means that there is a sub-sequence still denoted $\phi_{n}$ which converges strongly in $L^{2}(\Omega)$ and almost everywhere. For this sub-sequence $\left(\sigma\left(u_{n}\right) \phi_{n} \nabla \phi_{n}, \nabla w\right) \rightarrow$ $(\sigma(u) \phi \nabla \phi, \nabla w)$ for all $w \in H_{0}^{1}(\Omega)$. Since $\phi_{n} \rightarrow \phi$ in $L^{2}(\Omega)$ we also have $\left(f \phi_{n}, w\right) \rightarrow(f \phi, w)$ for all $w \in H_{0}^{1}(\Omega)$. Together we have,

$$
\begin{aligned}
& \left(f \phi_{n}, w\right)-\left(\phi_{n} \sigma\left(u_{n}\right) \nabla \phi_{n}, \nabla w\right) \rightarrow(f \phi, w)-(\phi \sigma(u) \nabla \phi, \nabla w), \\
& \quad \text { for all } w \in H_{0}^{1}(\Omega) .
\end{aligned}
$$

On the other hand we know that the sequence $\bar{u}_{n}$ associated with $u_{n}$ and $\phi_{n}$ is bounded in $H_{0}^{1}(\Omega)$ i.e. $\bar{u}_{n} \rightarrow y$ weakly in $H_{0}^{1}(\Omega)$ to some $y \in H_{0}^{1}(\Omega)$ i.e.,

$$
\left(\nabla \bar{u}_{n}, \nabla w\right) \rightarrow(\nabla y, \nabla w), \quad \text { for all } w \in H_{0}^{1}(\Omega) .
$$

Since $\bar{u}$ is the unique solution to (2.13) we conclude, by combining (2.15) and (2.16), that $y=\bar{u}$ i.e., $\bar{u}_{n} \rightarrow \bar{u}$ weakly in $H_{0}^{1}(\Omega)$. Since for any sub-sequence $u_{n}$, $\tilde{T} u_{n}=\bar{u}_{n}$ has a convergent sub-sequence we conclude that $\tilde{T} u_{n} \rightarrow T u$ for the entire sequence $u_{n}$. Since $\tilde{T}$ maps into $H_{0}^{1}(\Omega)$ which is compactly imbedded in $L^{2}(\Omega)$ we have that $T u_{n} \rightarrow T u$ strongly in $L^{2}(\Omega)$ and therefore in $S$ because of the a priori bound on $T u$. This means that $T$ is a continuous map from $S$ into itself.

\section{Discretization}

We are now going to discretize the weak forms (2.9) and (2.10). We start by introducing some notations. We let $\mathcal{K}_{h}=\{K\}$ be a set of disjoint elements such that $\cup K=\Omega$. We let $h_{K}$ denote the diameter of element $K$ and we further let $h=\max _{K \in \mathcal{K}_{h}} h_{K} \in \mathbf{R}^{+}$be the mesh size. We let $V_{h} \subset H_{0}^{1}(\Omega)$ be the set of piecewise linear basis functions on the mesh $\mathcal{K}_{h}$. We also introduce a nodal interpolation operator $\pi_{h}: C_{0}(\Omega) \rightarrow V_{h}$, where $C_{0}(\Omega)$ is the set of continuous functions on $\Omega$ that fulfills homogeneous Dirichlet boundary conditions. Note that $\left\|\pi_{h} v\right\|_{L^{\infty}(\Omega)} \leq\|v\|_{L^{\infty}(\Omega)}$ for all $v \in C_{0}(\Omega)$.

In order to establish $L^{\infty}(\Omega)$ bounds on the discrete approximation of $\phi$ that are independent of the mesh size, we need to make further assumptions on the mesh $\mathcal{K}_{h}$. We use the definition presented in [6]. We let $\left\{\varphi_{i}\right\}_{i \in \mathcal{N}}$ be the piecewise linear continuous basis functions which spans $V_{h}$. Furthermore we let $A$ be the stiffness matrix with entries $a_{i, j}=\left(\sigma\left(u_{h}\right) \nabla \varphi_{i}, \nabla \varphi_{j}\right)$. We say that two nodes $i$ and $j$ are adjacent if there is an edge $e_{i, j}$ connecting them. We assume the off-diagonal terms in $a_{i, j}$, 
corresponding to adjacent nodes $i$ and $j$, satisfies,

$$
\text { (A2) } a_{i, j} \leq-\frac{\rho}{h^{2}} \sum_{e_{i, j} \subset K}|K|, \quad \text { with } \rho>0 \text {, }
$$

where we sum over elements $K$ which shares the adjacent edge $e_{i, j}$. Such meshes can be constructed in three dimensions. We give an example from [6] in the numerical section below.

In the convergence proofs we are going to work with families of finite element meshes that approximates $H_{0}^{1}(\Omega)$. Each member of such a family $\left\{V_{h}\right\}$ will be associated with a mesh $\mathcal{K}_{h}$ fulfilling assumption (A2). Each member will also have a corresponding mesh size $h$. The elements in the family will be richer and richer in the sense that $h$ will decrease monotonically.

\subsection{The finite element method}

The finite element method now reads, find $\phi_{h} \in V_{h}$ and $u_{h} \in V_{h}$ such that,

$$
\begin{aligned}
\left(\sigma\left(u_{h}\right) \nabla \phi_{h}, \nabla v\right)=(f, v), & \text { for all } v \in V_{h}, \\
\left(\nabla u_{h}, \nabla w\right)=\left(f \phi_{h}, w\right)-\left(\phi_{h} \sigma\left(u_{h}\right) \nabla \phi_{h}, \nabla w\right), & \text { for all } w \in V_{h} .
\end{aligned}
$$

We are going to prove existence of solution to this system using Brouwder's fixed point theorem. First we need to show that $\phi_{h}$ is bounded independently of $h$ under the additional conditions on the meshes $\mathcal{K}_{h}$, (A2).

\subsection{Max norm estimate of the discrete potential}

When solving non-linear problems numerically it is often crucial to have a max norm estimate of the numerical solution. We state the following lemma.

Lemma 3.1 Under assumption (A2) the finite element approximation that solves (3.2) for any given $u_{h} \in V_{h}$ is bounded independently of the mesh size $h$, i.e. $\left\|\phi_{h}\right\|_{L^{\infty}(\Omega)} \leq C$, where $C$ is independent of $h$.

Proof See Sect. 6.2 in [6].

\subsection{Existence of discrete solution}

As mentioned above the proof will be based on Brouwder's fixed point theorem.

Theorem 3.1 Under the assumptions (A1) and (A2) there exists at least one solution $\left(\phi_{h}, u_{h}\right) \in V_{h} \times V_{h}$ to (3.2) and (3.3).

Proof We construct a fixed point map $\bar{u}_{h}=T_{h} u_{h}$, in analogy with the continuous case, given $u_{h} \in V_{h}$ let, $\phi_{h} \in V_{h}$ solve,

$$
\left(\sigma\left(u_{h}\right) \nabla \phi_{h}, \nabla v\right)=(f, v), \quad \text { for all } v \in V_{h} .
$$


Then we let $\bar{u}_{h} \in V_{h}$ solve,

$$
\left(\nabla \bar{u}_{h}, \nabla w\right)=\left(f \phi_{h}, w\right)-\left(\phi_{h} \sigma\left(u_{h}\right) \nabla \phi_{h}, \nabla w\right), \quad \text { for all } w \in V_{h} .
$$

Both these problems have unique solutions due to the Lax-Milgram and furthermore $\left\|\bar{u}_{h}\right\|_{H_{0}^{1}(\Omega)} \leq C$, where $C$ is depends on $\Omega$ but is independent of $h$ and the right hand side of (3.5), thanks to the max norm estimate in Lemma 3.1. This means that $T_{h}$ : $u_{h} \rightarrow \bar{u}_{h}$ maps $u_{h}$ into a closed ball. We repeat the arguments in the last paragraph of the proof of Lemma 2.3 and conclude that $T_{h}$ is continuous and therefore we can apply Brouwder's fixed point theorem which implies the existence of a solution to the discrete system (3.2) and (3.3).

We have thus proven the existence of a continuous solution pair $[\phi, u]$ to the system (2.9)-(2.10) and discrete solutions $\left[\phi_{h}, u_{h}\right]$ to the system (3.2)-(3.3). The next step is to show that the discrete solutions converge to a continuous solution as the mesh size $h \rightarrow 0$.

\section{Convergence of finite element solution}

In this section we prove that the numerical solution $\left[\phi_{h}, u_{h}\right]$ converges to a weak solution $[\phi, u]$ as $h \rightarrow 0$.

Theorem 4.1 Assume assumptions (A1) holds. Let $\left\{V_{h}\right\}$ be a sequence of finite element spaces fulfilling assumption (A2) with corresponding mesh sizes $\{h\}$, that decrease monotonically, and discrete solutions $\left\{\left[\phi_{h}, u_{h}\right]\right\}$. There exists a sub-sequence still denoted $\left\{\left[\phi_{h}, u_{h}\right]\right\}$ that converges to the weak solution in the following sense,

$$
\begin{aligned}
& \phi_{h} \rightarrow \phi \quad \text { in } H_{0}^{1}(\Omega) \cap L^{\infty}(\Omega) \quad \text { as } h \rightarrow 0, \\
& u_{h} \rightarrow u \quad \text { in } H_{0}^{1}(\Omega) \quad \text { as } h \rightarrow 0 .
\end{aligned}
$$

Proof The first part of the proof of this theorem follows ideas from the proof of Theorem 4.2 in [4]. We let $C_{0}^{\infty}(\Omega)$ denote all smooth functions that fulfill homogeneous Dirichlet boundary conditions. For any function $v \in C_{0}^{\infty}(\Omega)$ we have that $\pi_{h} v \in V_{h}$. This means that,

$$
\begin{aligned}
& \left(\sigma\left(u_{h}\right) \nabla \phi_{h}, \nabla \pi_{h} v\right)=\left(f, \pi_{h} v\right), \quad \text { for all } v \in C_{0}^{\infty}(\Omega), \\
& \left(\nabla u_{h}, \nabla \pi_{h} w\right)=\left(f \phi_{h}, \pi_{h} w\right)-\left(\phi_{h} \sigma\left(u_{h}\right) \nabla \phi_{h}, \nabla \pi_{h} w\right), \\
& \quad \text { for all } w \in C_{0}^{\infty}(\Omega) .
\end{aligned}
$$

The finite element solution proved to exist satisfies (4.3) and (4.4) and moreover $\left\|\phi_{h}\right\|_{H_{0}^{1}(\Omega)} \leq C,\left\|\phi_{h}\right\|_{L^{\infty}(\Omega)} \leq C$, and $\left\|u_{h}\right\|_{H_{0}^{1}(\Omega)} \leq C$. This means that a subsequence, still denoted $\phi_{h}$, converges $\phi_{h} \rightarrow \eta$ weakly in $H_{0}^{1}(\Omega)$, for some $\eta \in H_{0}^{1}(\Omega)$ and also that a sub-sequence, still denoted, $u_{h} \rightarrow y$ weakly in $H_{0}^{1}(\Omega)$, for some $y \in H_{0}^{1}(\Omega)$, as $h \rightarrow 0$. We also have $\pi_{h} v \rightarrow v$ and $\pi_{h} w \rightarrow w$ in $W^{1, \infty}(\Omega)$ as 
$h \rightarrow 0$, for all $v, w \in C_{0}^{\infty}(\Omega)$. The Dominated Convergence Theorem gives that $\sigma\left(u_{h}\right) \nabla \pi_{h} v \rightarrow \sigma(y) \nabla v$ up to a sub-sequence in $L^{2}(\Omega)$. We conclude,

$$
(\sigma(y) \nabla \eta, \nabla v)=(f, v), \quad \text { for all } v \in C_{0}^{\infty}(\Omega) .
$$

Since $C_{0}^{\infty}(\Omega)$ is dense in $H_{0}^{1}(\Omega)$ we have,

$$
(\sigma(y) \nabla \eta, \nabla v)=(f, v), \quad \text { for all } v \in H_{0}^{1}(\Omega) .
$$

We have thus showed that the limit to which a sub-sequence $\phi_{h} \rightarrow \eta$ weakly in $H_{0}^{1}(\Omega)$ solves an equation of the same type as (2.9). Next we show that the subsequence also converges strongly. For the weakly convergent sub-sequence $\phi_{h}$ we have,

$$
\begin{aligned}
\alpha\left\|\nabla\left(\eta-\phi_{h}\right)\right\|_{L^{2}(\Omega)}^{2} \leq & \left(\sigma\left(u_{h}\right) \nabla\left(\eta-\phi_{h}\right), \nabla\left(\eta-\phi_{h}\right)\right) \\
= & \left(\sigma\left(u_{h}\right) \nabla \phi_{h}, \nabla \phi_{h}\right)-2\left(\sigma\left(u_{h}\right) \nabla \eta, \nabla \phi_{h}\right) \\
& +\left(\sigma\left(u_{h}\right) \nabla \eta \nabla \eta\right) \\
= & \mathrm{I}+\mathrm{II}+\mathrm{III} .
\end{aligned}
$$

Since $\left[\phi_{h}, u_{h}\right]$ fulfills (3.2) we immediately have $\mathrm{I}=\left(f, \phi_{h}\right) \rightarrow(f, \eta)=$ $(\sigma(y) \nabla \eta, \nabla \eta)$ as $h \rightarrow 0$. Since $\phi_{h} \rightarrow \eta$ weakly in $H_{0}^{1}(\Omega)$ and for a sub-sequence $\sigma\left(u_{h}\right) \nabla \phi_{h} \rightarrow \sigma(\eta) \nabla y$ in $L^{2}(\Omega)$ by the Dominated Convergence Theorem II $\rightarrow$ $-2(\sigma(u) \nabla \eta, \nabla \eta)$. Finally, III $\rightarrow(\sigma(y) \nabla \eta, \nabla \eta)$ i.e. $\left\|\nabla\left(\phi_{h}-\eta\right)\right\|_{L^{2}(\Omega)} \rightarrow 0$ as $h \rightarrow 0$. We apply the Poincare-Friedrich inequality to conclude that there is a subsequence $\phi_{h} \rightarrow \eta$ strongly in $H_{0}^{1}(\Omega)$.

Next we turn to the second (4.4). Since a sub-sequence $\phi_{h} \rightarrow \eta$ strongly in $H_{0}^{1}(\Omega)$ there is a sub-sequence of this sequence that converges almost everywhere. For this sub-sequence still denoted $\phi_{h}$ the right hand side of (4.4) converges in the following sense,

$$
\begin{aligned}
& \left(f \phi_{h}, \pi_{h} w\right)-\left(\phi_{h} \sigma\left(u_{h}\right) \nabla \phi_{h}, \nabla \pi_{h} w\right) \rightarrow(f \eta, w)-(\eta \sigma(y) \nabla \eta, \nabla w), \\
& \quad \text { for all } w \in H_{0}^{1}(\Omega) .
\end{aligned}
$$

The first part is true since $\phi_{h} \rightarrow \eta$ strongly in $L^{2}(\Omega), \pi_{h} w \rightarrow w$ strongly in $L^{2}(\Omega)$, and $f \in L^{\infty}(\Omega)$. The second part is true since $\phi_{h} \rightarrow \eta$ almost everywhere and strongly in $H_{0}^{1}(\Omega)$ and $\sigma\left(u_{h}\right) \nabla \pi_{h} w \rightarrow \sigma(y) \nabla w$ in $L^{2}(\Omega)$. Since $u_{h} \rightarrow y$ weakly in $H_{0}^{1}(\Omega)$ we immediately have that the left hand side of (4.4) converges, $\left(\nabla u_{h}, \nabla \pi_{h} w\right) \rightarrow(\nabla y, \nabla w)$. This means that,

$$
(\nabla y, \nabla w)=(f \eta, w)-(\eta \sigma(y) \nabla \eta, \nabla w), \quad \text { for all } w \in H_{0}^{1}(\Omega),
$$

i.e. $(\eta, y)$ solves the system (2.9) and (2.10) which means that a sub-sequence of $\phi_{h} \rightarrow \phi$ strongly in $H_{0}^{1}(\Omega)$ and $u_{h} \rightarrow u$ weakly in $H_{0}^{1}(\Omega)$, where $(\phi, u)$ is a solution to (2.9) and (2.10). 
Finally, we prove that a sub-sequence $u_{h} \rightarrow u$ strongly in $H_{0}^{1}(\Omega)$. We start with $\left\|\nabla\left(u-u_{h}\right)\right\|_{L^{2}(\Omega)}^{2}$,

$$
\begin{aligned}
\left\|\nabla\left(u-u_{h}\right)\right\|_{L^{2}(\Omega)}^{2}= & \|\nabla u\|_{L^{2}(\Omega)}^{2}-2\left(\nabla u, \nabla u_{h}\right)+\left(\nabla u_{h}, \nabla u_{h}\right) \\
= & \|\nabla u\|_{L^{2}(\Omega)}^{2}-2\left(\nabla u, \nabla u_{h}\right)+\left(f \phi_{h}, u_{h}\right) \\
& -\left(\phi_{h} \sigma\left(u_{h}\right) \nabla \phi_{h}, \nabla u_{h}\right) \\
= & \mathrm{I}+\mathrm{II}+\mathrm{III}+\mathrm{IV} .
\end{aligned}
$$

Since $u_{h} \rightarrow u$ weakly in $H_{0}^{1}(\Omega)$ we immediately have I + II $\rightarrow-\|\nabla u\|_{L^{2}(\Omega)}^{2}$. Since $\phi_{h} \rightarrow \phi$ strongly in $L^{2}(\Omega), u_{h} \rightarrow u$ weakly in $L^{2}(\Omega)$, and $f \in L^{\infty}(\Omega)$, $\mathrm{III} \rightarrow(f \phi, u)$. Since $u_{h} \rightarrow u$ weakly in $H_{0}^{1}(\Omega)$ there is a sub-sequence still denote $u_{h}$ such that $u_{h} \rightarrow u$ almost everywhere. Since $\nabla \phi_{h} \rightarrow \nabla \phi$ in $L^{2}(\Omega)$ and $\sigma\left(u_{h}\right)$ is bounded and depends on $u_{h}$ continuously, the dominated convergence theorem gives us $\sigma\left(u_{h}\right) \nabla \phi_{h} \rightarrow \sigma(u) \nabla \phi$ in $L^{2}(\Omega)$. We also have $\phi_{h} \rightarrow \phi$ almost everywhere and $u_{h} \rightarrow u$ weakly in $H_{0}^{1}(\Omega)$ i.e. IV $\rightarrow-(\phi \sigma(u) \nabla \phi \nabla u)$ and therefore III + IV $\rightarrow$ $(f \phi, u)-(\phi \sigma(u) \nabla \phi \nabla u)=(\nabla u, \nabla u)$ which means that I + II + III + IV $\rightarrow 0$ as $h \rightarrow 0$. Again we use the Poincare-Friedrich inequality to conclude that $u_{h} \rightarrow u$ strongly in $H_{0}^{1}(\Omega)$ up to a sub-sequence.

Remark 4.1 Under the assumptions made in the paper the result presented in Theorem 4.1 is optimal (up to a subsequence). We only assume $H^{1}(\Omega) \cap L^{\infty}(\Omega) \times H_{0}^{1}(\Omega)$ regularity and we prove convergence of the numerical method under reasonable assumptions on the mesh and only Lipschitz boundary.

One could make stronger assumptions on the domain and data in order to derive an error bound. If we assume that there exist a solution such that $\phi, u \in H^{2}(\Omega) \cap H_{0}^{1}(\Omega)$, and that the linearization of the problem around the exact solution is Lipschitz continuous and has bounded inverse, we expect to get $h$ convergence in $H^{1}$-norm if the initial mesh is fine enough, see e.g. Theorem 2.6 in [10]. This can be seen in the first numerical example in Sect. 5.2, Table 1.

\section{Numerical examples}

We present two numerical examples. The first one shows that the solution converges linearly to a reference solution on a convex domain with smooth data. In the second example we present a more realistic engineering application on a more complicated geometry.

\subsection{Numerical methods}

The finite element approximation defined by (3.2) and (3.3) is computed iteratively according to the following Gauss-Seidel like procedure, starting with $u_{h}^{0}=0$ : 
1. Update $\sigma$, using recently computed $u_{h}^{n}$.

2. Solve (3.2) to obtain $\phi_{h}^{n+1}$.

3. Solve (3.3) to obtain $u_{h}^{n+1}$ by evaluation the right hand side in $\phi_{h}^{n+1}$ and $u_{h}^{n}$.

4. Repeat steps 1-3 until $\left\|u_{h}^{n+1}-u_{h}^{n}\right\|_{H_{0}^{1}(\Omega)}+\left\|\phi_{h}^{n+1}-\phi_{h}^{n}\right\|_{H_{0}^{1}(\Omega)}<t o l$, where tol is a given parameter specifying the error tolerance.

In the performed numerical examples we use $t o l=10^{-6}$. Equations (3.2) and (3.3) are solved individually on a tetrahedral mesh, $\mathcal{K}_{h}$, using continuous piecewise linear basis functions.

\subsection{Convergence study}

We study the convergence in energy norm using a structured tetrahedral mesh as described in [6]. The mesh is constructed by dividing the domain into equally sized cubes, with each cube consisting of five tetrahedrons. As our domain, $\Omega$, we used the unit cube in three dimensions. A picture of the mesh, consisting of four cubes in each coordinate direction is shown in Fig. 1. In order for these meshes to be conforming, the smaller cubes need to be partitioned into tetrahedrons in two different ways, see Fig. 2. It can be shown that this construction fulfills assumption (A2) by studying the local stiffness matrix, see [6] and further [11]. To study the energy norm convergence, a reference solution on a mesh with 128 cubes in each coordinate direction and compared with solutions computed on meshes with $4,8,16,32$, and 64 cubes in each coordinate direction.

We let $f=10 x y z e^{-\left((x-0.5)^{2}+(y-0.5)^{2}+(z-0.5)^{2}\right)}$ and $\sigma(u)=0.05+\frac{0.15}{\pi}\left(\frac{\pi}{2}+\right.$ $\left.\arctan \left(\frac{u+0.05}{0.05}\right)\right)$ and plot the solution in Figs. 3-4 and the convergence in Fig. 5.

Fig. 1 Structured mesh with $4 \times 4$ cubes/face
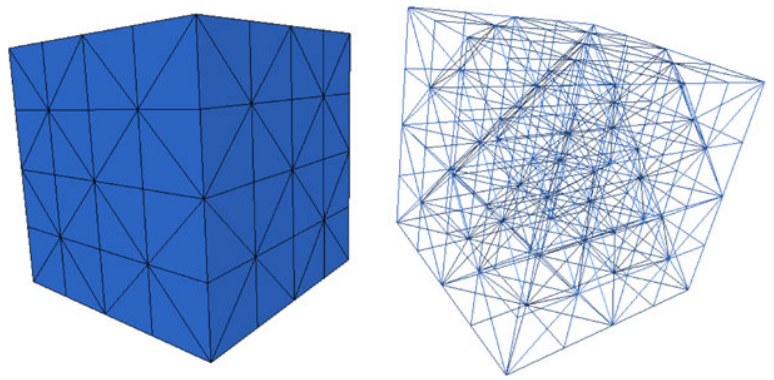

Fig. 2 The two different partitions needed in order to make the mesh conforming
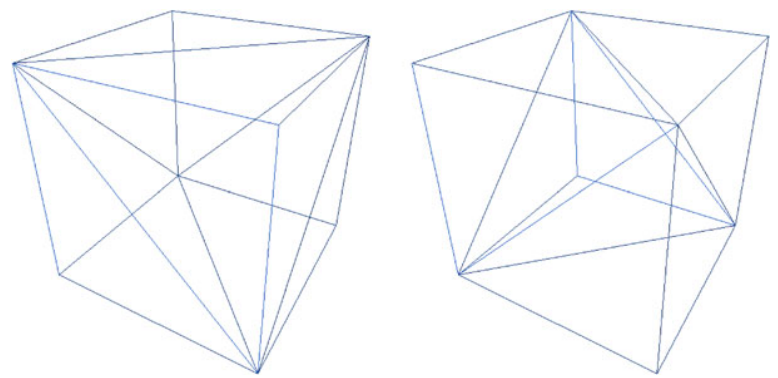

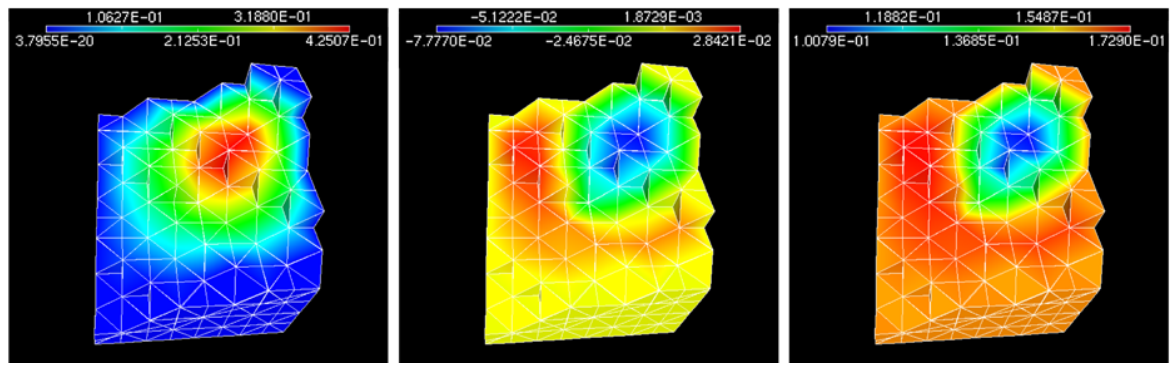

Fig. $3 \phi(\mathbf{a}), u(\mathbf{b})$, and $\sigma(\mathbf{c})$ with $8 \times 8$ cubes/face
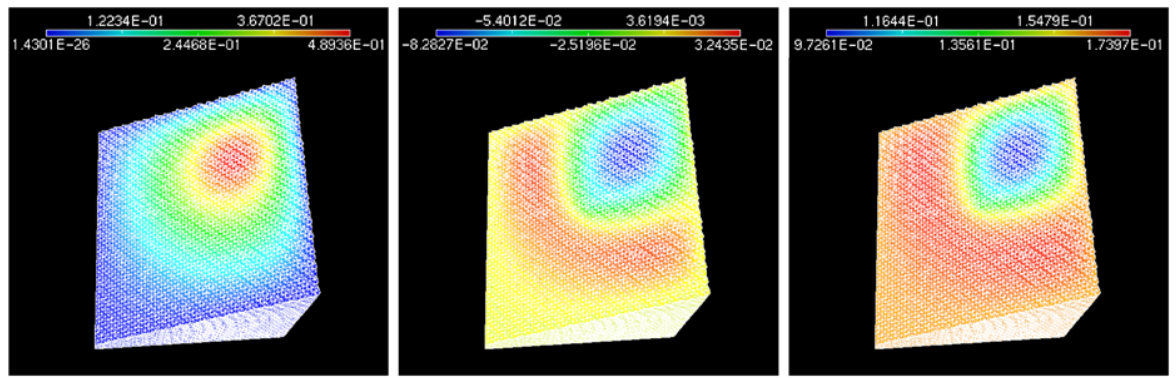

Fig. $4 \phi(\mathbf{a}), u(\mathbf{b})$, and $\sigma$ (c) with $64 \times 64$ cubes/face
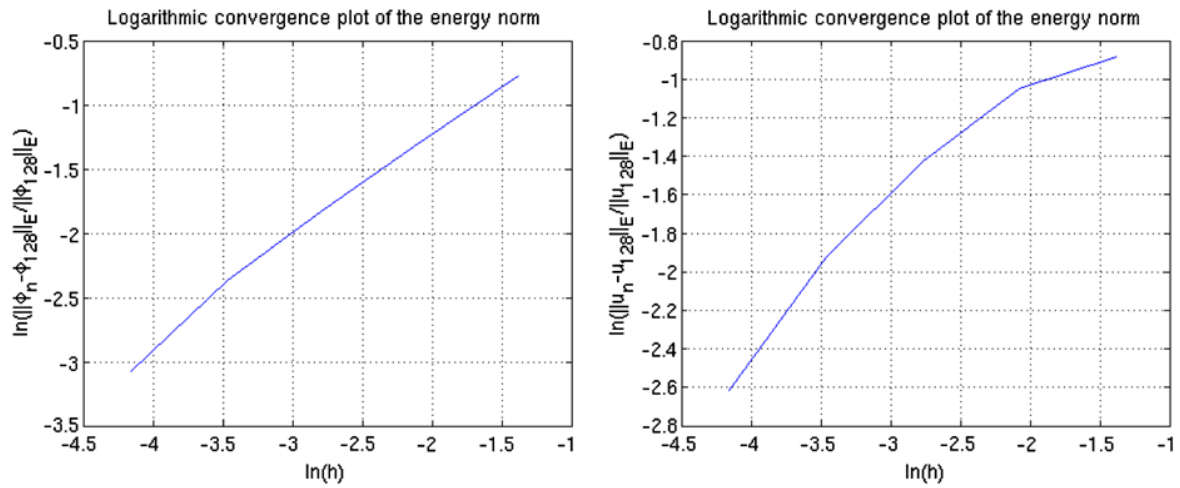

Fig. 5 A logarithmic plot of the relative error in energy norm as a function of $h$

Studying Fig. 5 the convergence rate seems to approach approximately 1 which is what would be expected on a convex domain with smooth data. The values of the relative errors in the energy norm can also be found in Table 1.

The numerical values of the entries in the stiffness matrix $a_{i, j}=\left(\sigma\left(u_{h}\right) \nabla \varphi_{i} \nabla \varphi_{j}\right)$ fulfilled assumption (A2). 
Table 1 The relative error in energy norm of $u_{h}$ and $\phi_{h}$ as a function of $h$

\begin{tabular}{lll}
\hline$h$ & Relative error in $\phi_{h}$ & Relative error in $u_{h}$ \\
\hline 0.25 & 0.460 & 0.413 \\
0.125 & 0.276 & 0.350 \\
0.0625 & 0.164 & 0.234 \\
0.03125 & 0.094 & 0.146 \\
0.015625 & 0.046 & 0.073 \\
\hline
\end{tabular}

\subsection{Engineering application}

In this section we provide solutions obtained on a more complicated geometry. We have chosen to solve the equations on a MEMS device (Micro Electro Mechanical System) (see e.g. [9]). This will force us to diverge a little from the main theory of the paper. We will not construct the mesh so that it fulfills (A2), since this becomes very technical on a complicated geometry. We will also use more complicated boundary conditions. We still use the method we have presented in the paper. The theoretical analysis need to cover the worst case regularity. In real applications the situation is usually more regular then that. This is why we still expect the method to give good results.

A picture of the geometry is found in Fig. 6. This particular device is designed to work as an electrical switch by applying a potential difference between the two pads. This gives rise to an electrical current and due to the construction of the device, heat development will be larger in the upper more "narrow" part of the geometry. This will cause the device to bend, thus making it possible to close an electrical circuit. To fully simulate this requires the incorporation of the equation for thermal expansion, which we have not included. The domain has been meshed with a standard tetrahedral mesh with linear basis functions. The natural boundary conditions to apply to this problem are mixed boundary conditions. We have used:

$$
\begin{aligned}
& \phi=1, \quad y=0 \quad \text { and } \quad z>0 \\
& \phi=-1, \quad y=0 \quad \text { and } \quad z<0 \\
& \boldsymbol{n} \cdot \nabla \phi=0, \quad y \neq 0 \\
& u=0, \quad y=0 \\
& \boldsymbol{n} \cdot \nabla u=0, \quad y \neq 0
\end{aligned}
$$

These boundary conditions corresponds to a potential difference between the two pads at $(y=0, z>0)$ and $(y=0, z<0)$, as well as fixing the temperature at the pads. The solutions obtained with these boundary conditions, using $f=0$ and $\sigma(u)=$ $0.5+\frac{1.5}{\pi}\left(\frac{\pi}{2}+\arctan \left(\frac{u-0.25}{0.005}\right)\right)$ are shown in Fig. 7 .

Remark 5.1 As mentioned above, the boundary condition used in the second example are different from the ones used in the mathematical framework. The mixed boundary condition for the electrical potential equation will not affect the conclusion that 
Fig. 6 Picture of the geometry. The outer measurements are $11 \times 192 \times 29$
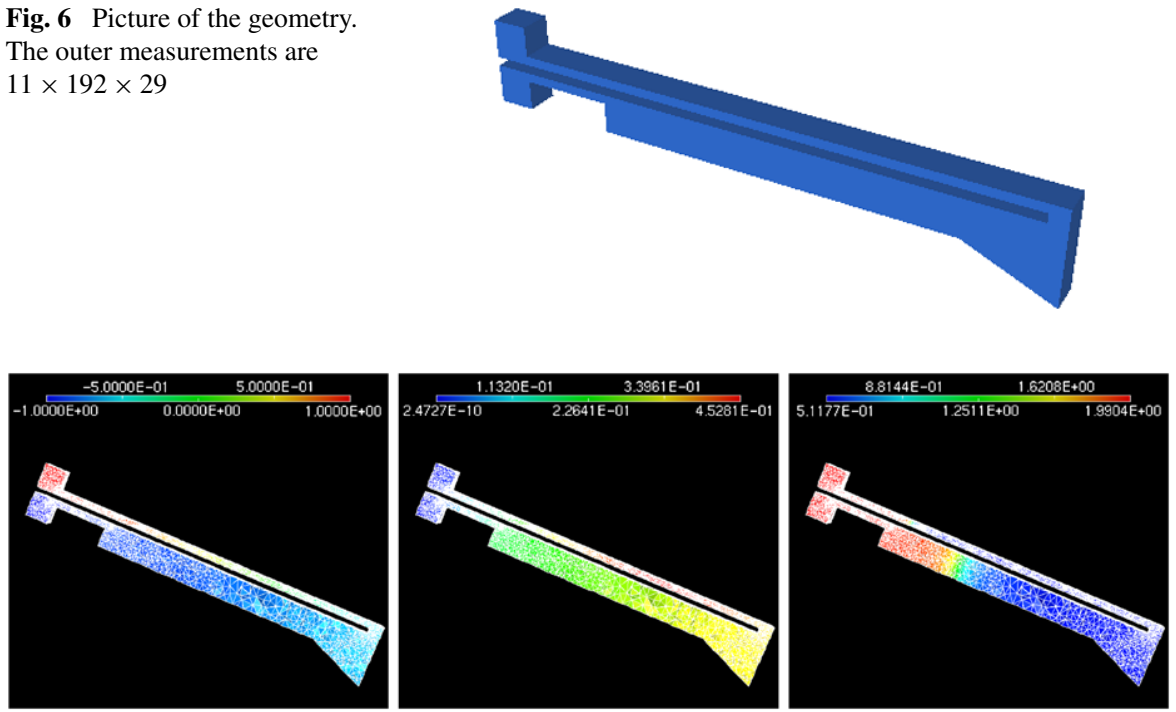

Fig. $7 \phi(\mathbf{a}), u(\mathbf{b})$, and $\sigma(\mathbf{c})$ on the MEMS-geometry

$\phi \in H^{1}(\Omega) \cap L^{\infty}(\Omega)$. However, the trick in (1.2) can not immediately be applied in the temperature equation unless Dirichlet boundary conditions are used since test the functions are now in a larger space.

Open Access This article is distributed under the terms of the Creative Commons Attribution Noncommercial License which permits any noncommercial use, distribution, and reproduction in any medium, provided the original author(s) and source are credited.

\section{References}

1. Adams, R.A.: Sobolev Spaces. Academic Press, San Diego (1978)

2. Akrivis, G., Larsson, S.: Linearly implicit finite element methods for the time-dependent Joule heating problem. BIT 45, 429-442 (2005)

3. Boccardo, L., Orsina, L., Poretta, A.: Existence of finite energy solutions for elliptic systems with $L^{1}$ valued nonlinearities. Math. Models Methods Appl. Sci. 18(5), 669-687 (2008)

4. Bradji, A., Herbin, R.: Discretization of the coupled heat and electrical diffusion problems by the finite element and the finite volume methods. IMA J. Numer. Anal. 28(3), 469-495 (2008)

5. Casado-Díaz, J., Chacón Rebollo, T., Girault, V., Mármol Gómez, M., Murat, F.: Finite elements approximation of second order linear elliptic equations in divergence form with right-hand side in $L^{1}$. Numer. Math. 105(3), 337-374 (2007)

6. Chen, L., Holst, M., Xu, J.: The finite element approximation of the nonlinear Poisson-Boltzmann equation. SIAM J. Numer. Anal. 45(6), 2298-2320 (2007)

7. Elliott, C.M., Larsson, S.: A finite element model for the time-dependent Joule heating problem. Math. Comput. 64, 1433-1453 (1995)

8. Gallouët, T., Herbin, R.: Existence of a solution to a coupled elliptic system. Appl. Math. Lett. 7(2), 49-55 (1994)

9. Henneken, V.A., Tichem, M., Sarro, P.M.: In-package MEMS-based thermal actuators for microassembly. J. Micromech. Microeng. 16(6), 107-115 (2006)

10. Holst, M.J., Tsogtgerel, G., Zhu, Y.: Local convergence of adaptive methods for nonlinear partial differential equations. arXiv:1001.1382v1 
11. Kerkhoven, T., Jerome, J.W.: $L_{\infty}$ stability of finite element approximations to elliptic gradient equations. Numer. Math. 57, 561-575 (1990)

12. Stampacchia, G.: L probléme de Dirichlet pour les équations elliptiques du second ordre á coefficients discontinus. Ann. Inst. Fourier (Grenoble) 15(1), 189-258 (1965)

13. Sun-Sig, B.: Elliptic equations with BMO coefficients in Lipschitz domains. Trans. Am. Math. Soc. 357(3), 1025-1046 (2004) 